\title{
Use of a Simple Non-Destructive Technique for Evaluation of the Elastic and Vibration Properties of Fiber-Reinforced and 3D Fiber-Metal Laminate Composites
}

\author{
Davide De Cicco and Farid Taheri * \\ Advanced Composite and Mechanics Laboratory, Department of Mechanical Engineering, Dalhousie University, \\ 1360 Barrington Street, P.O. Box 15 000, Halifax, NS B3H 4R2, Canada; davide.decicco@dal.ca \\ * Correspondence: farid.taheri@dal.ca; Tel.: +1-902-494-3935; Fax: +1-902-484-6635
}

Received: 1 December 2017; Accepted: 28 February 2018; Published: 2 March 2018

\begin{abstract}
The aim of this paper is to assess the accuracy and reliability of a simple non-destructive sonic technique for evaluating the effective elastic and vibration properties (damping coefficient) of various isotropic and orthotropic materials-in particular, of a recently developed class of 3D fiber-metal laminates (FML). Aluminum, E-glass/epoxy composite, 3D-FML, and glass-reinforced aluminum FML (GLARE) materials were considered. It is exhibited that the 3D-FML offers the greatest damping characteristics in comparison to all the considered materials. Moreover, the sonic technique, facilitated through the use of a GrindoSonic equipment, proves to produce accurate and reliable results with minimal effort. Finite element analysis is also employed to further establish the accuracy of the properties evaluated by the experimental data. The utility of the established homogenized experimental properties within the finite element framework is also discussed.
\end{abstract}

Keywords: vibration; damping; fiber-metal laminate; GrindoSonic; non-destructive testing

\section{Introduction}

Fiber reinforced composite materials (i.e., axial particulates embedded in fitting matrices), are commended for their remarkable specific strength and stiffness and non-corrosive nature. Therefore, they are widely used in a variety of applications in construction [1], aerospace [2], automotive [3], sporting and consumer goods [4], and in medical and dental applications, with either net [5] or long [6] fibers. However, the hereinabove mentioned properties are not the only beneficial properties of this class of material; they also exhibit excellent vibration damping characteristics compared to bulk metals amongst many other positive attributes.

Vibration analysis of composite materials, either in the configuration of laminates or sandwich, have long been the focus of a great number of analytical, numerical, and experimental research. From the perspective of dynamic response analysis, in the early 1970s, Noor [7] highlighted limitations of the available analytical models for the evaluation of low frequency vibration of simply supported thick composite beams. Since then, several investigators have expended efforts in developing analytical models for accurate evaluation of the natural frequency of composites [8-10]. For instance, Kant and Swaminathan [11] developed a higher-order theory for free vibration analysis of sandwich plates, accounting for the through-thickness shear and strain/stress effects, thus, leading to more accurate modeling of the cross sections warping. Tu et al. [12] developed a finite element formulation for a nine-node rectangular element accounting for the rotary inertia effects and the parabolic variation of the through-thickness shear. They conducted various parametric studies to demonstrate the effectiveness of the method in predicting the vibration response of composite and sandwich plates. For more insight 
into the analytical and numerical methods available for free vibration analysis of composite materials, the reader is referred to a comprehensive literature review presented by Sayyad and Ghugal [13].

Enhancing the dynamic damping properties of laminated composite and sandwich structural elements has also been vigorously investigated by several researchers. The use of nanoparticles, naturally damped materials, and external damping sources have been identified as the most common and effective enhancement methods. For instance, the benefits of including a small amount of carbon nanotubes (CNTs) has been investigated by various researchers [14,15]. Khan et al. [16] demonstrated experimentally that an increase in the damping ratio of carbon-reinforced composite polymers could be attained by addition of CNTs, and that a higher particle content leads to higher damping values. They also highlighted the fact that the improvement of their composite's performance in its totality was mainly due to the improvement of the damping properties of composite's matrix. DeValve and Pitchumani [17] gave further insight into the damping effect of CNTs inclusion in a matrix by modeling the particles/matrix interaction. It was reported that the addition of 1-2 wt \% of particles led to a $40-60 \%$ improvement in damping property. Damping enhancement at low frequencies can also be controlled by using piezoelectric patches, as well as by taking advantage of the viscoelastic properties of materials for enhancing material damping at the higher vibration frequencies. Araújo et al. [18] successfully carried out numerical simulations by using ABAQUS finite element software to demonstrate the effectiveness of the mentioned approach. On the other hand, Trindade et al. [19] developed an analytical model, which was linked into a finite element code, to account for the effect of damping gained using piezoelectric patches, as well as accounting for the viscoelastic response of the host material.

The use of inherently damped natural materials as an effective means for improving damping characteristics of various structural components has also been demonstrated by various researchers. For instance, Sargianis et al. [20] reported a 100\% increase in the structural damping ratio of a sandwich beam formed by using balsa wood as its core and natural fibers forming the facial laminated layers. The same researchers attained further improvements in damping response (as great as 233\%) by incorporating Rohacell ${ }^{\circledR} 51$ WF synthetic core instead of the balsa core in the mentioned sandwich composite. It should be noted that the mentioned enhancement was accomplished with a marginal loss in the overall flexural rigidity of the sandwich composites compared to the sandwich beams with glass laminates as face sheets and synthetic core. Prabhakaran et al. [21] also reported improvements in the structural damping property of a laminate fiber-reinforced epoxy composite when flex fibers were used instead of glass fibers. The investigators reported an improvement in the acoustic damping properties of the composite, an attribute that plays an important role in some application, such as vehicles' shell. Other investigators have also discussed about the existing link between the structural and acoustic damping ratios [22,23].

The 3D fiber-metal laminate (3D-FML) developed by our research group falls into the category of materials that are considered as suitable candidates in forming vehicles' panels, mainly due to its excellent crashworthiness properties [24,25], as well as its favorable vibration characteristics that will be introduced in this article. Specifically, the damping characteristics of this recently developed FML will be investigated and will be compared to that of glass-reinforced aluminum (GLARE), the most widely used FML.

It should be noted that accurate evaluation of vibration characteristics of materials can be a time-consuming task, requiring a certain degree of expertise. Various investigators have used impulse excitation technique for establishing materials damping response. For instance, Cheraghi et al. [26] used piezoelectric sensors to record vibration response of Polyvinyl chloride (PVC) pipes and evaluated the material's damping coefficient. The investigators discussed the utility of various methods that could be used to extract the damping coefficient from the vibration data. The logarithmic decrement analysis, the Hilbert transform analysis, the Hilbert-Huang spectral analysis, and the Empirical Mode decomposition method were the methods investigated by Cheraghi et al. Naghipour et al. [27] proposed a modification of the half-power bandwidth method to account for the high damping effect. They investigated damping response of glue-laminated beams (glulam) reinforced with fiberglass/epoxy 
composite interlayers. The results of the experimental tests showed that their proposed modifications could produce improved values compared to three methods that are conventionally used to obtain damping coefficient of materials. Therefore, we will also examine the utility of the above-mentioned acoustic method for evaluating the vibration and damping response of materials.

Moreover, elastic properties of a material could also be obtained from vibration data and analysis. The applicability of the dynamic excitation technique for characterization of elastic properties of composites was demonstrated by Viens and Johnson [28] in the NASA technical memorandum 104629. The authors of that report used the GrindoSonic testing equipment [29] to obtain the elastic modulus of composite beams and showed that adequate agreement compared to the reference measurements (i.e., $\pm 5 \%$ with respect to the average value) could be obtained. Zou et al. [30] used the same equipment to obtain the natural frequency of glue-laminated timber beams and evaluated the material's equivalent modulus of elasticity, demonstrating the reliability of the method. Similar to the vibration technique, Droz et al. [31] also used a guided wave-based method to obtain both the tensile and shear moduli of sandwich panels. Transition bandwidth and Hilbert transform were used for processing the data. They also proposed a set of procedures to speed up post-processing of the data. It is worth mentioning that various non-destructive techniques have been used for detecting damage in fiber reinforced composites in various fields [32].

In summary, the excellent vibration properties of composites make them a material of choice in applications where both vibration and weight are the design constraints. A prominent example of such an application is outer-body automobile parts, where they are subjected to various types of loading and vibration. In addition, today, fuel economy and safety standards are additional constraints that vehicles designers are challenged with. An attempt to overcome such challenges led to the development of a truly three-dimensional fiber-metal laminate (3D-FML) introduced in this article. This FML consists of 3D fiberglass fabric (3D-FGF), which has several hollow cavities through its thickness, which could be filled with a foam for added stiffness. This stiff and highly damped composite is sandwiched between thin sheets of a magnesium alloy. This hybrid composite system offers an excellent response under both static and low-velocity impact loadings [24,25,33,34]. However, its vibration characteristics have not been explored yet, especially how its vibration properties differ compared to the conventionally used FML, GLARE [35], as well as other light-weight materials such as aluminum and fiberglass-reinforced epoxy.

Therefore, this paper presents the viability and reliability of a simple acoustic technique, facilitated through the GrindoSonic equipment, for establishing elastic and vibration properties of various isotropic and orthotropic materials, including the complex hybrid composite fiber-metal laminates briefly introduced above.

\section{Materials and Methods}

\subsection{Materials}

The 6061-T6 aluminum was obtained from a local vendor, while the $0.5 \mathrm{~mm}$ thick AZ31B-H24 magnesium alloy sheets were acquired from MetalMart International (Commerce, CA, USA). The three-dimensional fabric was obtained from China Beihai Fiberglass Co. (Jiujiang City, Jiangxi, China) and the unidirectional fabric from Vectorply (Phenix City, AL, USA). The epoxy resin, composed of two parts (i.e., bisphenol-A-based Araldite LY 1564 resin and Aradur 2954 (cycloaliphatic polyamine) hardener), was obtained from Huntsman Co. (West Point, GA, USA). A two-part, 8-lb density urethane foam was supplied by US Composites (West Palm Beach, FL, USA). Finally, the GLARE specimens were provided to us by Professor Laliberté of Carlton University (Ottawa, ON, Canada). 


\subsection{Specimen Manufacturing}

The 3D-FML and fiber-reinforced plastic (FRP) panels were fabricated in situ. Three beam-shape specimens were extracted from each material type. The specimens were $200 \mathrm{~mm}$ long, with width of $20 \mathrm{~mm}$, while their thickness would vary based on the material type.

The acquired GLARE panels had a thickness of $1.4 \mathrm{~mm}$. Aluminum specimens were machined to the dimensions from a 3.64 mm-thick 6061-T6 aluminum panel. The FRP specimens were extracted from a panel consisting of 12 layers of unidirectional fiberglass fabric with a layup configuration of $(0 / 0 / 90 / 0 / 90 / 0)_{\mathrm{s}}$, resulting in a total thickness of $4.8 \mathrm{~mm}$. The panel was fabricated using the resin infusion technique, so as to ensure uniform resin content. After the infusion process, the panel was kept under vacuum and cured at $60^{\circ} \mathrm{C}$ for two hours, then at $120^{\circ} \mathrm{C}$ for eight hours.

The 3D-FML were fabricated following the procedure described in detail in [36]; the procedure is briefly summarized here. First, the 3D fiberglass fabric (3D-FGF) was impregnated with epoxy resin and cured under the same regime as followed in fabricating the FRP panel. Then, polyurethane foam was aspired into the hollow structure of the 3D-FGF and left to harden for at least $24 \mathrm{~h}$. Lastly, the magnesium skins, whose surfaces were roughened by grit-blasting, cleaned with acetone, and air-dried, were bonded to the core using the same epoxy used in the fabrication of the glass fabrics. The entire assembly was cured under vacuum to ensure optimal bond among the constituents. The final thickness of the 3D-FML panel was measured as $5.4 \mathrm{~mm}$. Figure 1 shows the 3D-FML in its three main fabrication phases.

It should be noted that in the case of aluminum specimens, the portion of the specimen that would come in contact with the GrindoSonic probe, was slightly abraded to generate minor roughness with the purpose of preventing the slippage of the probe during the vibratory motion of the beam. This was not necessary for the other specimens because the cutting procedure (used for extraction of the specimens from the panels) naturally induced rugosities.

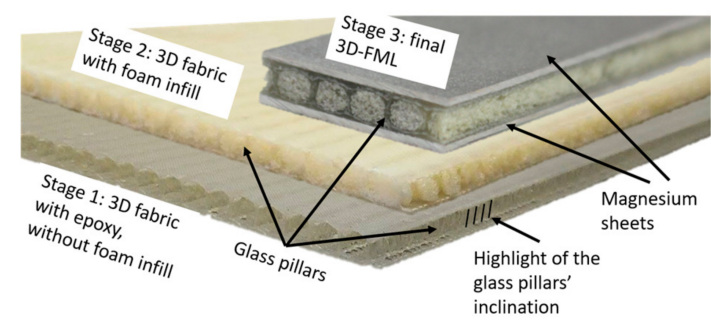

(a)

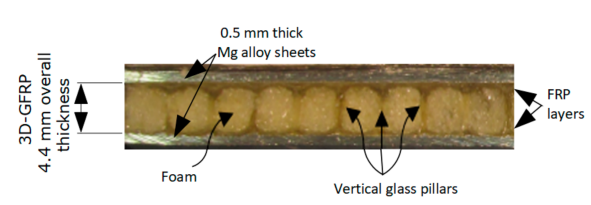

(b)

Figure 1. The three-dimensional fiber-metal laminate (3D-FML) specimen: (a) its fabrication phases; and $(\mathbf{b})$ its constituents and dimensions.

\subsection{Testing and Analysis Procedures}

The experimental apparatus for obtaining the vibration data, namely the GrindoSonic, is shown in Figure 2a, while the setup for determining the bending stiffness of the specimens is presented in Figure $2 \mathrm{~b}$. For the vibration analysis, the specimens were supported by two prismatically-shaped low-density foam to simulate the free-free boundary conditions. As mentioned in both the equipment manufacturer user-manual and NASA technical memorandum 104629 [28], a light hammer with a stiff tip is to be used for exciting the specimens. Two excitation hammers were fabricated: one consisting of an aluminum ball attached to a thin aluminum rod, and the second one consisting of a steel ball attached to a thin wooden rod. Based on the preliminary tests, the steel-wood hammer proved to produce more consistent excitations in all specimen types. Therefore, all results reported in this paper were obtained by exciting the specimens with the steel-wood hammer.

The equipment includes a small, highly-sensitive probe, which is put into contact with the specimen at a short distance from one of the vibration-free nodes for detecting the oscillatory movement of a given specimen. It outputs a change in the electric potential proportional to 
the amplitude of the vibration generated by the oscillatory motion of the specimen. The signal is acquired by a GrindoSonic Model MK5i equipment (J.W. Lemmens, Leuven, Belgium), which automatically analyzes the transient response of the vibratory motion of the excited specimen and computes the frequency.

The natural frequency of the material is immediately and directly displayed by the GrindoSonic once a test (excitation) has been conducted. However, to be able to extract the damping ratio of a given material, one needs to capture the entire vibratory motion occurring as a result of the excitation. Therefore, the GrindoSonic was connected to a data-acquisition system and the National Instruments' Signal Express software was used to acquire and store the entire vibration data (using a sampling rate of $100 \mathrm{kHz}$ ). The information was later processed using a code written in LabVIEW environment, as will be described in Section 2.5 .

To minimize the vibration measurement errors, the foam supports should be positioned at the vibration nodes of a given specimen (i.e., where the vibration amplitude is null). The location's distance, $s$, measured from each end of the specimen, which is associated with the fundamental flexural vibration mode of a homogeneous beam, can be established by the following equation [37]:

$$
s=0.224 L
$$

where $L$ is the beam length. However, because most of the specimens used in the study are not homogeneous, finite element eigenvalue analysis was conducted to verify the position of the nodes for those specimens. It was shown that, for all specimens, the nodes were located at approximately $45 \mathrm{~mm}$ form each specimen's end, thus matching the analytical predictions. In addition, the results of our preliminary tests revealed that a misplacement of the support, even with a relatively large margin of $10-\mathrm{mm}$ distance from the nodes, did not affect the measured frequencies. Therefore, the distance of $45 \mathrm{~mm}$ (measured from each end) was used for all specimens. Lastly, it is worth mentioning that each beam examined in this study was excited a minimum of seven times to obtain accurate average results.

The bending stiffness of each specimen was evaluated by conducting a 3-point bending test using a MTS servo-hydraulic testing machine, equipped with digital electronic controller. The specimens were simply supported over a span of $190 \mathrm{~mm}$ and loaded at their mid-span quasi-statically until a mid-span deflection of $4 \mathrm{~mm}$ was reached. The force-deflection curve was directly recorded by the testing machine. Exception was made when testing the GLARE specimens because of their relatively very low thickness. Therefore, GLARE specimens were supported at a 90-mm span.

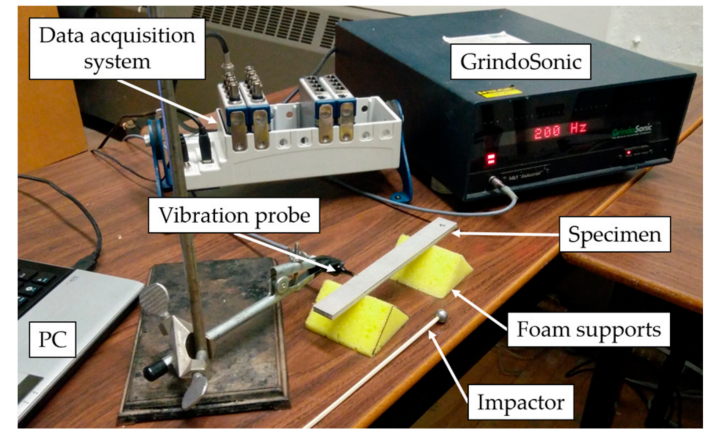

(a)

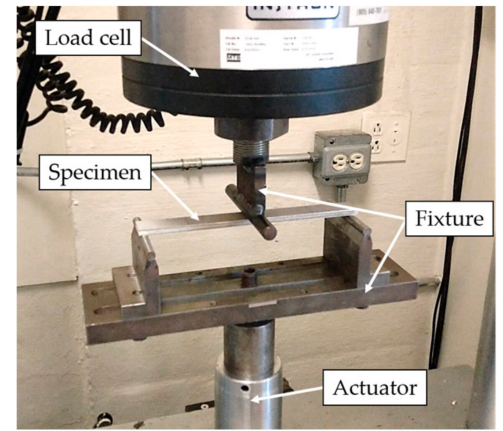

(b)

Figure 2. (a) Vibration test setup and the apparatus; and (b) 3-point bending test setup.

\subsection{Theoretical Basis for Evaluation of the Frequency and Damping Coefficient}

\subsubsection{Frequency}

The frequency displayed by the GrindoSonic for each material type will be compared to frequency obtained from finite element analysis (FEA) and the available closed-form solution. Details regarding 
the finite element simulations will be provided in Section 2.6. The analytical equation is obtained following the procedure described by Kelly [38], synthetized in this section. The governing equation of motion for a prismatic beam, free of loading, is as follows:

$$
\mu \frac{\partial^{2} v}{\partial t^{2}}+E I \frac{\partial^{4} v}{\partial x^{4}}=0
$$

where $v$ is the deflection of the beam, $t$ is the time, $x$ is the position along beam's span, $\mu$ is the mass per unit length, $E$ is the modulus of elasticity, and $I$ is the second moment of inertia of the cross-section. Considering only the stationary solution, the deflection can be expressed by the product of functions of the independent variables:

$$
v(x, t)=X(x) T(t)
$$

where $X(x)$ and $T(t)$ represent the parts of the solution for the deflection that depend only from the position along the beam and the time, respectively. They can be represented by the following mathematical expressions:

$$
\left\{\begin{array}{l}
X(x)=C_{1} \sin \left(\frac{\gamma}{L} x\right)+C_{2} \cos \left(\frac{\gamma}{L} x\right)+C_{3} \sinh \left(\frac{\gamma}{L} x\right)+C_{4} \cosh \left(\frac{\gamma}{L} x\right) \\
T(t)=D_{1} \cos (2 \pi f t)+D_{2} \sin (2 \pi f t)
\end{array}\right.
$$

where $f$ is the vibration frequency, $\mu=2 \pi f L^{2} \sqrt{\mu /(E I)}$, and $C_{1, \ldots, 4}$ and $D_{1,2}$ are constants determined using the boundary conditions. Limiting our study to free-free boundary conditions leads to the following analytical expression for evalulation the flexural frequency of the beam:

$$
f_{n}=\frac{1}{2 \pi}\left(\frac{\gamma_{n}}{L}\right)^{2} \sqrt{\frac{E I}{\mu}}
$$

where $f_{\mathrm{n}}$ is the $n$th vibration mode, $L$ is the length of the beam, and $\gamma_{n}$ is a coefficient found numerically from the following equation, resulting from the solution of Equation (2), using Equation (3) and the free-free boundary conditions:

$$
\cos \gamma \cosh \gamma=1
$$

The above function has many roots; since we are only interested in the fundamental flexural frequency of the beam in this study, only the first value of $\gamma_{n}$ is computed (i.e., $\gamma_{n}=4.73$ ). Moreover, in order to apply the equation to laminated composite beams, the bending stiffness for a homogeneous beam, $E I$, is replaced by $b D_{11}$, where $b$ is the width of the beam, and $D_{11}$ is the bending stiffness per unit width of the laminate in the longitudinal direction. This leads to the following form of the equation used to theoretically estimate the fundamental flexural frequency $\left(f_{T H}\right)$ of a beam with a constant cross section, under free-free boundary conditions:

$$
f_{T H}=\frac{1}{2 \pi}\left(\frac{4.73}{L}\right)^{2} \sqrt{\frac{R}{\mu}}, \text { with } R=\left\{\begin{array}{l}
E I \text { for homogeneous beams } \\
b D_{11} \text { for composite beams. }
\end{array}\right.
$$

\subsubsection{Damping Coefficient}

Materials' damping characteristics plays an important role in controlling the response of structures subject to vibratory loading. The knowledge of damping coefficient enables the designers to simplify the need for otherwise complicated set of calculations. In other words, designers can convert a complex vibration analysis into a much simpler quasi-static analysis by amplifying the time-dependent loading applied to a given structure by knowing the value of material damping coefficient. Therefore, evaluation of this parameter is of paramount importance. Notwithstanding, the conventional methods of analysis for determining the damping coefficient are time-consuming, as it has been highlighted by Naghipour et al. [27], where they discuss various techniques for evaluating the damping properties of 
E-glass fiber-reinforced glue-laminated beams. Therefore, identification of a more robust and simplified approach has been of interest to the authors [26,30]. In addition, identification of a material that could offer optimal damping has also been a fundamental research objective to the authors for the past few years. Therefore, the methodologies followed to meet the objectives are briefly outlined here.

A time-dependent signal, $x(t)$, representative of the damped vibratory motion of a system consists of an amplitude, $A$, varying sinusoidally over time. Given that the damping ratio of the system, $\xi$, is lower than the critical value $\xi=1$, as it is the case in our study, the amplitude will decrease following an exponential curve, referred to as the envelope of the signal. A general representation of the signal and its envelope is given in Figure 3. Mathematically, the signal can be written as:

$$
x(t)=A e^{-\xi \omega_{0} t} \cos \left(\omega_{D} t-\phi\right),
$$

where and $\omega_{D}$ is the damped angular frequency of the system and $\phi$ is the phase angle (not relevant to our analysis). The following relationship can be used to relate the damped natural frequency to the undamped frequency, $\omega_{0}$ :

$$
\omega_{D}=\omega_{0} \sqrt{1-\xi^{2}} .
$$

Moreover, the terms appearing within the brackets in Equation (8) represent the envelope (i.e., the upper and lower bounds) of the signal. Therefore, it is possible to extract the damping coefficient from the signal by using the decremental logarithm technique $(\delta)$, over an oscillation period, $T_{D}$, defined as the ratio between two consecutive maxima of the signal starting at the $i$ th oscillation (see Figure 3). Mathematically, $\delta$ can be presented by the following expression:

$$
\delta=\ln \frac{x_{i}}{x_{i+1}} .
$$

Combining Equations (8) and (10) yields the explicit relationship between the decremental logarithm and the damping coefficient, as:

$$
\delta_{i}=\ln \frac{A e^{-\tau \omega_{0} t_{i}} \cos \left(\omega_{D} t-\phi\right)}{A e^{-\tau \omega_{0}\left(t_{i}+T_{D}\right)} \cos \left(\omega_{D} t-\phi\right)} .
$$

Thus, given that $T=2 \pi / \omega_{D}$, and combining Equation (11) with Equation (8), one obtains the expression for calculating the damping coefficient:

$$
\xi=\frac{\delta_{i}}{\sqrt{4 \pi^{2}+\delta_{i}^{2}}} .
$$

Since the evaluation of the decremental logarithm will be done using the experimental data, one could attain more accuracy by conducting the evaluation over multiple oscillation periods instead of a single one. Therefore, a modified version of the decremental logarithm will be used in this study, which can be represented mathematically as:

$$
\delta_{i}=\frac{1}{n} \ln \frac{x_{i}}{x_{i+1}},
$$

where $n$ refers to the number of considered periods. Subsequently, one can evaluate the damping parameter by using the following expression:

$$
\xi=\left\langle\frac{\delta_{i}}{\sqrt{4 \pi^{2}+\delta_{i}^{2}}}\right\rangle,
$$


the mathematical operator $\langle\cdot\rangle$ referring to the average of the quantity $\cdot$ The extraction of the decremental logarithm and calculation of the damping coefficient were automated via the use of an algorithm written in LabVIEW environment, as will be explained in the next section.

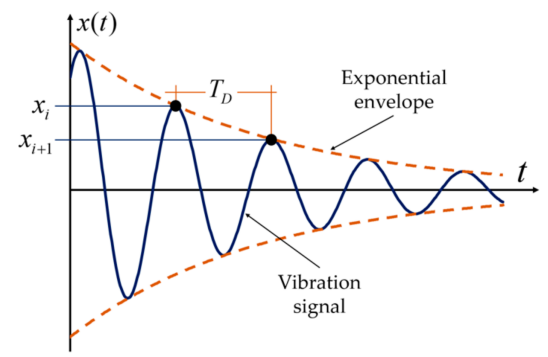

Figure 3. Illustration of the fundamental elements for vibratory analysis.

\subsection{Vibratory Data Extraction and Analysis}

In this section, the procedure used to retrieve the desired information from the vibratory data obtained with the GrindoSonic is reported. For the sake of clarity, a summary of the procedure is provided in the flowchart illustrated in Figure 4. As mentioned above, a simple experimental method to obtain the natural frequency of a beam is achieved by using the GrindoSonic testing equipment. The equipment software automatically calculates the time elapsed between two amplitude peaks of a vibration signal, starting from the time that corresponds to a decrease in signal's intensity, and outputs the frequency. The second methodology used to extract the natural frequency entailed the use of the entire vibration event data obtained for each tested specimen. More specifically, this was done by obtaining the power spectrum of the signal using LabVIEW's Spectral Measurements subroutine. This subroutine takes the signal produced by GrindoSonic as its input, performs a Fast Fourier Transform (FFT) of the signal, and outputs the amplitude for each frequency contained within the signal. Figure 5 illustrates a typical power spectrum of the signals corresponding to the FRP and 3D-FML specimens. As can be seen, the natural frequency is clearly visible from the graphs (i.e., the ones with the greatest amplitude). This frequency was then compared to the one obtained from the GrindoSonic, as will be reported in Section 3.1.

The second step of the analysis involved obtaining the signal envelope, from which the damping coefficient of the material could be calculated, for which an algorithm was developed in LabVIEW. First, a bandpass filter of $\pm 30 \mathrm{~Hz}$ was applied to the portion of the signal in the vicinity of the natural frequency in order to remove most of the noise and obtain a smooth oscillating signal. Then, the envelope was extracted from the refined signal by incorporating the Hilbert transform of the signal, following the procedure described by Cheraghi et al. [26]. The Hilbert transform, $H(t)$, entails the following operation:

$$
H(t)=\frac{1}{\pi} \int_{-\infty}^{+\infty} u(\tau) \frac{x(\tau)}{t-\tau} d \tau
$$

In practical terms, the envelope is prescribed by the modulus of the complex number, whose real part contains the signal's amplitude, and its imaginary part includes the Hilbert transform of the signal. The extraction procedure was implemented in LabVIEW as per instructions provided by Yang et al. [39]. Typical results produced by the procedure are illustrated in Figure 6. In the figure, the signals for FRP and 3D-FML, as well as their respective envelopes, are illustrated.

Lastly, the damping coefficient was estimated. Using the envelope, an algorithm coded in LabVIEW calculates the decremental logarithm of the signal over 50 oscillations, for all available successive points within the envelope, and then outputs the average damping factor, $\xi$, computed by Equation (14). For each considered material, a cut-off value was designated, which established the period of time over which the decremental logarithm was calculated, so that a maximum number 
of tests could be analyzed with the same settings. For instance, since the damping coefficient of the aluminum alloy considered here is relatively very low compared to that of FRP and 3D-FML, a one-second signal duration could be considered for accurately extracting the material's damping coefficient. In comparison, only a $0.4 \mathrm{~s}$ long signal duration was used in calculating 3D-FML's damping coefficient, since this material has relatively much greater damping property. Notwithstanding, the intervention of the user was kept to a minimum in the process of isolating the portion of data that was included in the analysis, and for tuning the aforementioned parameters. The minimal user intervention contributes to reaching the maximum consistency and reliability of the results, as well as reducing the time required for the analysis to be performed.

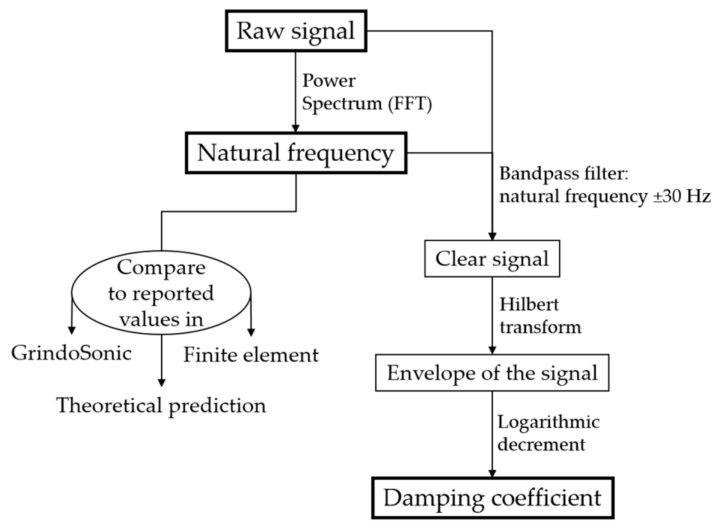

Figure 4. Algorithm used to analyze the oscillation data.

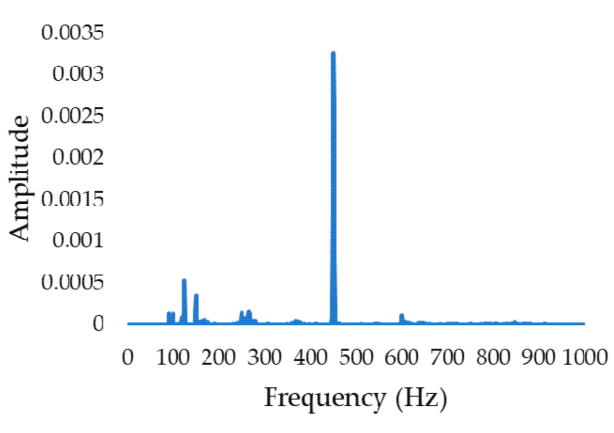

(a)

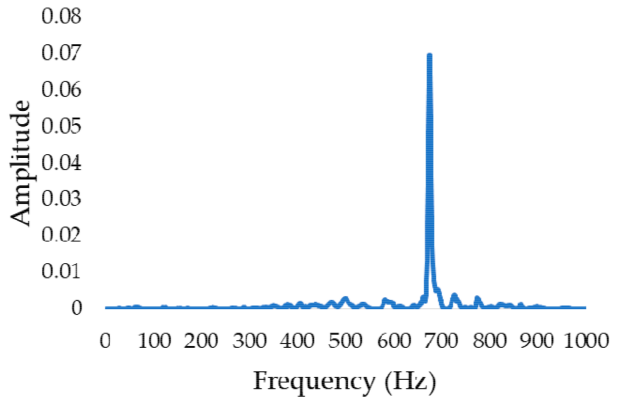

(b)

Figure 5. Power spectrum up to $1000 \mathrm{~Hz}$ for: (a) FRP (fiber-reinforced plastic); and (b) 3D-FML specimens.

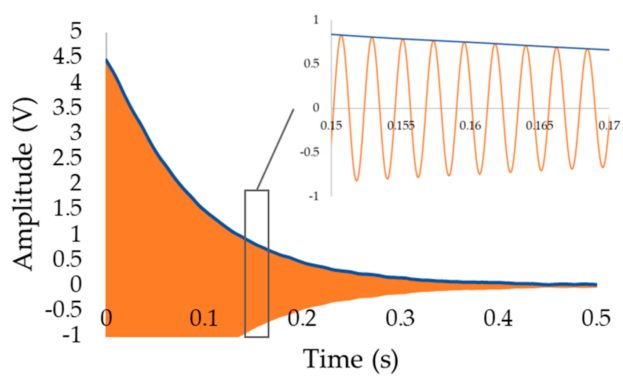

(a)

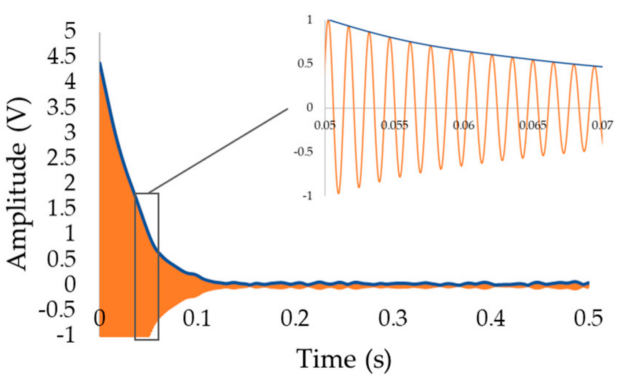

(b)

Figure 6. Representative vibration signals and envelopes for (a) the FRP and (b) 3D-FML specimens. 


\subsection{Finite Element Simulations}

Various finite element models were constructed using the commercial software LS-DYNA in order to simulate the response of the beam specimens considered in this study. The purpose of the simulations was twofold: (i) to evaluate the precise location of vibration nodes prior to performing the vibration tests; and/or (ii) to assess the usability of the extracted data by conducting a simple and efficient numerical analysis. The models can be divided into two categories: homogeneous beams and composite beams. All specimens were modeled using 8-node 3D isoparametric elements, with reduced integration (type 1 in the software). Linear eigenvalue analysis was conducted to obtain the natural frequency of each material type specimen. Convergence analyses were carried out to establish the optimal mesh refinement with the aim of establishing acceptable accuracy of the results, yet maintaining the computational efficiency (see Figure 7).

It should be noted that two approaches were used to analyze the FRP, 3D-FML, and GLARE beam specimens. The purpose was to determine whether one can attain accurate results by using the homogenized properties of the material systems, as opposed to modeling each ply or constituents individually, thereby minimizing the computation effort significantly. Therefore, in the first case, as was done for the case of aluminum beam specimens, the FRP, 3D-FML, and GLARE specimens were analyzed as homogenized beams by incorporating the homogenized properties that were established through the vibration frequency analysis $\left(f_{\mathrm{HOM}}\right)$. In other words, the various material layers and ply orientations were homogenized as a unique bulk material, represented by a unified modulus of elasticity and Poisson's ratio. In the second analysis scheme, all the plies were modeled using orthotropic properties (see the graphical representation in Figure 7b-d). More specifically, the 12 layers of the FRP specimens, and in the case of the 3D-FML specimens, the 3D fiberglass fabric, magnesium skins and foam core, and aluminum and fiberglass layers of the GLARE specimens were all modeled individually. Further, because linear eigenvalue analysis was performed, perfect bond among all constituents forming these specimens was assumed; in other words, no tie or contact algorithms were used to connect various layers to one another.

From the material modelling perspective, a distinction between isotropic and orthotropic behavior was made to account for the effect of material anisotropy when applicable. In other words, the actual material properties, calibrated via authors' previous works and reported in the Appendix A, were used for the simulations, so that a reference frequency, $f_{F E}$, could be obtained and used for comparison with the measured and analytically predicted frequencies.

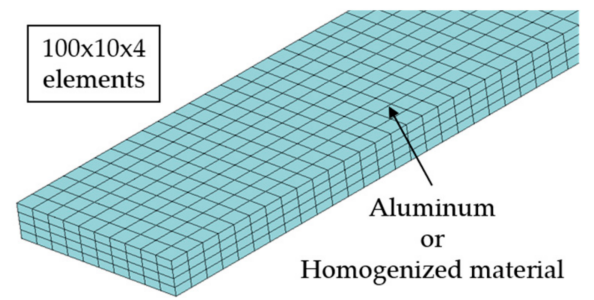

(a)

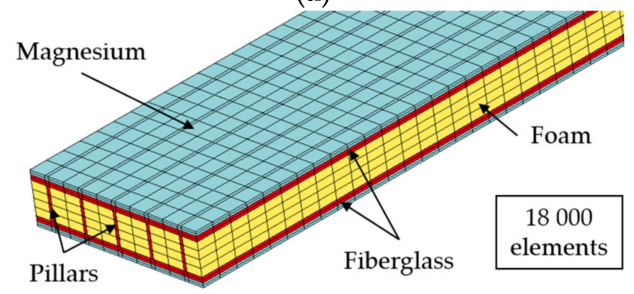

(c)

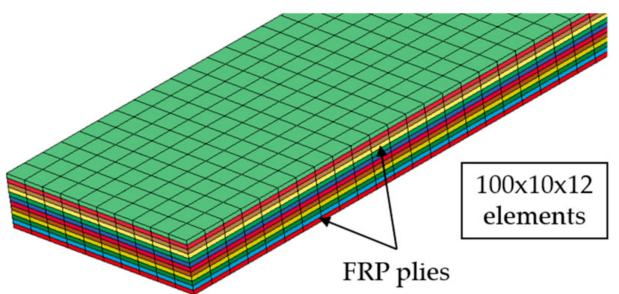

(b)

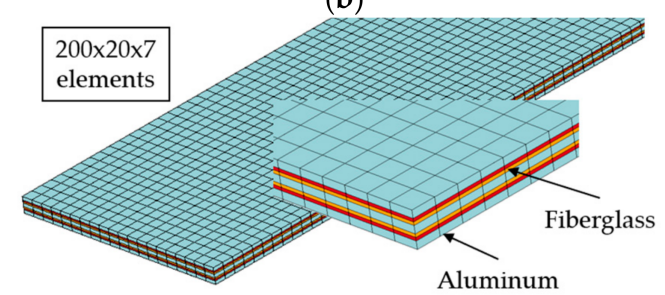

(d)

Figure 7. Mesh topologies used to analyze: (a) aluminum and homogenized beams; (b) FRP beam; (c) 3D-FML beam; and (d) glass-reinforced aluminum (GLARE) beam. 


\section{Results and Discussion}

The results obtained from the analyses are presented in this section. In addition, some practical guidelines are provided to assist the evaluation of the vibration properties of other composite materials in an efficient and accurate manner. Note that each reported result is the average of at least seven tests conducted on specimens (i.e., 21 tests per material category).

\subsection{Frequency Extraction}

The accuracy of the fundamental frequency obtained by the GrindoSonic has been demonstrated by various authors $[28,30,40]$. However, as was also mentioned in NASA technical memorandum 104629 [28], the GrindoSonic sometimes produces inaccurate values, especially in cases where: (i) excessive noise is generated by the user while imparting the excitation impulse to the specimen; and (ii) if the specimen is excited by tapping it at an incorrect location, which may cause the excitation of higher vibration modes of the specimen. The average values of the frequencies obtained from the GrindoSonic, for all tested materials, are reported in Table 1. Note that the standard deviation was found to be less than $0.6 \%$ of the mean values; therefore, it is not reported in the table. It should also be noted that the GrindoSonic could not detect the fundamental frequency of GLARE specimens, but rather revealed an average frequency of $1675 \mathrm{~Hz}$. This is close to the frequency corresponding to the 5th vibration mode of the material (i.e., $1654.49 \mathrm{~Hz}$ ), found using FEA (refer to Section 3.3). Moreover, the 1st vibration mode frequency of $184.87 \mathrm{~Hz}$ obtained from the FEA is very close to $183.68 \mathrm{~Hz}$ frequency obtained from the power spectrum analysis of the complete vibration signal captured through GrindoSonic. It is postulated that perhaps the relatively very small depth-to-span aspect ratio of GLARE specimens is responsible for the gross discrepancy observed in the case of GLARE specimens. To corroborate the hypothesis, three GLARE specimens were bonded together to generate a thicker specimen, resulting in a total thickness of $4.44 \mathrm{~mm}$. To ensure statistically reliability, a large number of trials (i.e., 18 trials) was conducted when exciting the specimens. The difference between the frequency displayed by the GrindoSonic and the one obtained using the power spectrum technique was decreased to a mere $0.12 \%$, thus validating our hypothesis.

Table 1. Natural frequency results obtained through GrindoSonic (GS). FRP: fiber-reinforced plastic; 3D-FML: three-dimensional fiber-metal laminate; GLARE: glass-reinforced aluminum.

\begin{tabular}{cccccc}
\hline & Aluminum & FRP & 3D-FML & GLARE & Thick GLARE \\
\hline GS vibration frequencies (Hz) & 462.61 & 450.62 & 681.49 & 183.68 ₹ & 544.73 \\
\hline $\begin{array}{c}\text { \% Difference in frequencies obtained } \\
\text { by GS and FFT of the signal }\end{array}$ & $<0.01 \%$ & $-0.04 \%$ & $-0.19 \%$ & $>80 \%$ & $-0.12 \%$ \\
\hline $\begin{array}{c}\% \text { Number of times incorrect } \\
\text { frequency was reported by GS }\end{array}$ & $48.9 \%$ & $2.8 \%$ & $14.3 \%$ & $100 \%$ & $0 \%$ \\
\hline
\end{tabular}

$¥$ Value reported for this material is obtained through post-processing (FFT) of GrindoSonic signal by LabVIEW.

Also reported in Table 1 are the percentages of the number of times erroneous values of the natural frequency were reported by the GrindoSonic, which could be due to the anomalies described above. In other words, the values represent the percentage of trials that the device's frequency reading did not match the frequency obtained by through post-processing (FFT) analysis of the same signal (in its totality). As can be seen, FRP specimens are the least sensitive (by a mere $2.8 \%$ discrepancy) to the anomalies that could affect the reported GrindoSonic's readings, follows by readings of 3D-FML (14.3\%), aluminum with $48.9 \%$, and lastly $100 \%$ discrepancy in the case of GLARE specimens, for the reason disclosed earlier. Neglecting this latter case (since the cause of the discrepancy was the low thickness-to-length ratio of the specimens), it appears that specimens having a greater metal content are more sensitive to noise. One may conclude that the precision of the results would be greater when GrindoSonic is used to evaluate the natural frequency response of composite materials in comparison to metallic materials. 


\subsection{Elastic Property Evaluation}

From the obtained frequencies, the equivalent modulus of elasticity of the specimens were calculated and reported in Table 2. The evaluation was done using GENEMOD, a software that accompanied the GrindoSonic. Based on the specimen dimensions, density (or mass), and fundamental vibration frequency, the software calculates and reports the equivalent elastic modulus. Note that a constant Poisson's ratio of 0.3 is considered by the software in the calculations. The software was originally developed based on formulation pertinent to homogeneous materials; therefore, material's inhomogeneity and anisotropy are not accounted for by the software. Thus, it is of interest to see its limitations in calculating the property of fiber-reinforced composites and even of the more complex hybrid composites, such as the 3D-FML considered in this study. For an unbiased comparison, the bending stiffness of the specimens was chosen as the comparison criterion. Therefore, the bending stiffness of the specimens evaluated through 3-point bending tests are compared to the bending stiffness values obtained using the equivalent modulus of elasticity reported by the software; the values are reported in Table 2. Excellent agreement is found in results of the aluminum and GLARE (with the maximum discrepancy of $0.48 \%$ ). Further, good agreement is observed when comparing the results for the FRP specimens (maximum discrepancy of 6.15\%). This discrepancy is attributed to the relatively complex internal structure of the FRP, which seems to affect the vibratory response of the material-therefore resulting in a larger apparent stiffness. This issue is also believed to be the cause of the slightly greater discrepancy of $16.51 \%$ observed in the case of 3D-FML. In general, flexural stiffness is affected by any inhomogeneity in materials (i.e., voids, fiber/resin ratio, etc.). The complex distribution of fibers in the 3D glass constituent of the 3D-FML is believed to be responsible for the greater comparative discrepancies. It should be noted that the percent of vertical fiber content (the fibers that connect the two bi-axial fabrics of the 3D fiberglass fabric) could vary by an appreciable percent from one specimen to another. This is due to the fact that the specimens have a relatively narrow width, and they may contain varying volume of resin or voids in the vicinity of each group of vertical fibers. In addition, the injected foam may include non-uniform voids within the small cavities of the 3D-FML, which can also affect the material damping, especially when narrow (small size) specimens are considered. Those anomalies could therefore affect the resulting the vibratory response and hence the apparent stiffness by a considerable margin. In addition, Schultz and Tsai [41] reported that, for unidirectional fiberglass beams, the elastic modulus of the material obtained from vibration analysis was observed to be greater than the value obtained from the appropriate static testing by as much as $27 \%$ and as low as $7 \%$. Therefore, it is believed that more accurate results could be obtained by accounting for the strain-rate dependency of the materials in the calculation of the equivalent elastic modulus using the natural vibration value.

Table 2. Comparison of the equivalent Young's modulus and bending stiffness obtained from GrindoSonic analysis and 3-point bending tests.

\begin{tabular}{ccccc}
\hline Material & $\begin{array}{c}\text { Equivalent Young's } \\
\text { Modulus (GPa) }\end{array}$ & $\begin{array}{c}\text { Experimental Bending } \\
\text { Stiffness }\left(\text { GPa-m }{ }^{4}\right)\end{array}$ & $\begin{array}{c}\text { GrindoSonic Bending } \\
\text { Stiffness }\left(\text { GPa-m }{ }^{4}\right)\end{array}$ & \% diff \\
\hline Aluminum & 66.02 & 5305.00 & 5306.75 & 0.03 \\
FRP & 22.77 & 3953.66 & 4196.97 & 6.15 \\
3D-FML & 21.73 & 4894.51 & 5702.82 & 16.51 \\
GLARE & 62.70 & 297.79 & 299.21 & 0.48 \\
\hline
\end{tabular}

\subsection{Integrity of the Results}

The integrity of the equivalent modulus of elasticity values obtained through GrindoSonic for all the tested materials was evaluated by using them as an input in FEA models, and calculating the resulting frequency. These resulting different frequencies were compared with those obtained by GrindoSonic $\left(f_{G S}\right)$ and the closed-form solution $\left(f_{T H}\right)$. The results are reported in Table 3, revealing that the GrindoSonic can capture the natural frequency of the tested specimens with good accuracy and reliability. Note that $f_{F E}$ is the frequency obtained by the finite element models for which properties of each constituent 
were provided explicitly, while $f_{H O M}$ refers to the model that used the homogenized elastic properties of the materials as its input (i.e., that obtained from the GrindoSonic, as reported in Table 2).

As seen in the case of aluminum, when the properties were provided explicitly, a maximum discrepancy of $0.42 \%$ was observed between the frequency values produced by GrindoSonic and FEA. On the other hand, when the homogenized elastic value (or the actual experimental value) was fed to the finite element code, the maximum discrepancy was reduced to $0.26 \%$. This further enforces the effectiveness of GrindoSonic use to obtain accurate and reliable data. As can also be seen, the results produced for the other tested materials reveal that in all cases, the use of homogenized elastic properties as an input to the FE models (i.e., those obtained through the GrindoSonic) produced results with fewer discrepancies in comparison with the cases when each material's elastic properties were fed to the software, explicitly. The results further confirm the reliability of this testing technique when considering composite and hybrid materials with complex material configurations.

Moreover, as was mentioned earlier, the GrindoSonic was not able to capture the vibration frequency of the 1st mode for the GLARE specimens, but rather displayed a frequency of around $1675 \mathrm{~Hz}$. Since the FEA results were demonstrated to be reliable, FEA was used to gain further understanding of the phenomenon that affects the frequency response of the GLARE considered in this study. In summary, as stated, the FE analysis predicted a vibration frequency of $1654.49 \mathrm{~Hz}$ for the 5th vibration mode. The complete vibration-history data, which was recorded by the GrindoSonic, was subsequently analyzed using a LabVIEW code. Processing of the signal revealed that a vast majority of the signal power was originated from a frequency around $184 \mathrm{~Hz}$, which corresponds to the natural frequency of the GLARE specimen. Thus, this latter value is taken as a reference value for the calculation of the material properties and the finite element simulations. Note that in practical situations, where one does not have access to the complete vibration data of GLARE, the equivalent properties of GLARE cannot be obtained.

It should also be mentioned that discrepancies in the $f_{F E}$ vibration values could also be attributed to the possible errors involved in each evaluated mechanical property. It is observed that the more complex a given hybrid material's configuration, the greater would be the impact of the properties on the analysis' results. For instance, in the case of 3D-FML, evaluation of the individual constituent's mechanical properties would be an onerous task. Therefore, one can appreciate the utility of GrindoSonic and the minimal effort required in evaluating the equivalent properties of such hybrid composites.

Moreover, comparison of the frequencies reveals another interesting phenomenon, which is that discrepancies between the predicted values (obtained either numerically or analytically) are higher in the case of specimens that include dissimilar interfaces (e.g., magnesium/fiberglass interfaces in the case of 3D-FML, and aluminum/fiberglass interfaces in the case of GLARE). Further, more dissimilar layers' configuration apparently adversely affects the results (though this could be associated to the existence of voids and other inhomogeneities in various constituents, as described earlier). It, however, seems plausible that the interfaces could influence the vibration behavior of the beams. Nevertheless, even though FRP is a composite material, it does not have any bi-material interface at the macro-scale; in other words, all the plies are fused together during the curing process; therefore, no interface, as such, is generated. Therefore, further investigation into the influence of the interfaces may be warranted for optimizing the vibration response of such hybrid composites.

Table 3. Comparison of the frequencies obtained experimentally (with GrindoSonic), numerically and analytically.

\begin{tabular}{ccccc}
\hline Material & $f_{G S}(\mathrm{~Hz})$ & $f_{F E}(\mathrm{~Hz}) / \%$ diff. & $f_{H O M}(\mathrm{~Hz}) / \%$ diff. & $f_{T H}(\mathrm{~Hz}) / \%$ diff. \\
\hline Aluminum & 462.61 & $460.67 / 0.42$ & $461.39 / 0.26$ & $462.46 / 0.03$ \\
FRP & 450.62 & $442.26 / 1.86$ & $446.99 / 0.81$ & $447.43 / 0.71$ \\
3D-FML & 681.49 & $626.71 / 8.04$ & $660.20 / 3.12$ & $631.20 / 7.38$ \\
GLARE & $183.68 \%$ & $184.87 /-0.65$ & $161.78 / 11.92$ & $190.59 /-3.76$ \\
\hline Value reported for this material is obtained through post-processing (FFT) of GrindoSonic signal.
\end{tabular}




\subsection{Damping Coefficients}

Another important material property that could be extracted from the vibration analysis is the damping coefficient of the materials. As expected, considering the foam-filled nature of the 3D-FML, this new hybrid composite material exhibited the best damping properties among the other materials. The damping response of 3D-FML is superior to that of aluminum, GLARE and FRP by $181 \%, 82 \%$, and $23 \%$, respectively. The immediate effect of the damping ratio on the global vibrational response is evidenced in the signals illustrated in Figure 8, where a comparison between the FRP and 3D-FML vibration responses are reported. As can be seen, the ratio of 1.2 in damping exhibited by 3D-FML in comparison to FRP leads to a sensitive difference in the behavior, and it can be clearly seen how rapidly vibration of the 3D-FML specimen is attenuated. This renders this hybrid material a suitable material for use in systems that would be subjected to continuous vibration (for example, transportation vehicles). In addition, the selection of the infilling foam can be optimized to further enhance the material's damping characteristic.

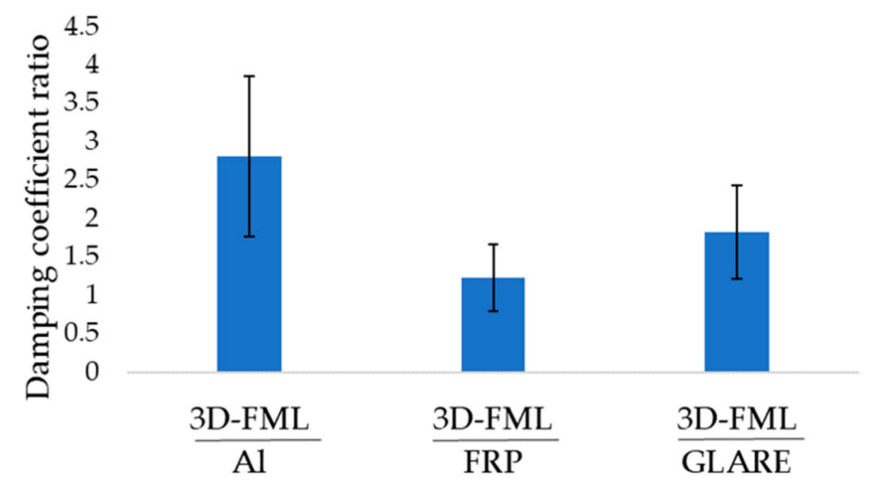

Figure 8. Normalized damping coefficient of the 3D-FML.

\subsection{Effect of the Probe's Position}

An investigation was carried out to examine the effect of location at which GrindoSonic's probe should be placed to attain most accurate readings. This issue takes importance when evaluating vibration characteristics of thick panels and panels made of dissimilar materials (such as the 3D-FML, and other sandwich composites). For that, tests were performed by varying the position of the probe along three different location within thickness of the panel (specifically, at locations corresponding to the elevations of the magnesium layer, FRP layer and mid-thickness of the core). Noting that a small variation in the recorded signal could be expected, to obtain acceptable statistical maximum reliability, 20 tests were conducted for measuring the signal at each location. Figure 9 shows the outcome of the tests. As can be seen, the measurements from the core produced the highest frequency, while the ones from the fiberglass layer produce the lowest; however, the percentage difference is of only $0.09 \%$, which is negligible. Moreover, the standard deviation obtained from core measurements is also relatively smaller. This can be attributed to the minimal chances of slippage of the probe on the measuring surface because of the porous nature of the foam. Therefore, it is recommended to take GrindoSonic's measurement at the core section for a maximum reliability in the results, and conducting a small number of tests.

\subsection{Effect of Specimen's Length}

Finally, the influence of specimen's length on the equivalent elastic modulus of the 3D-FML is studied by performing vibration tests on 3D-FML specimens with various lengths. In addition to the $200 \mathrm{~mm}$ specimens that were used for conducting all above-mentioned tests, specimens with $150 \mathrm{~mm}$ - and $100 \mathrm{~mm}$-lengths were also considered. As described in the previous sections, the elastic 
modulus of each specimen was evaluated by converting the natural frequency of the specimen obtained by the GrindoSonic equipment; the results are reported in Figure 10. Contrary to what would be expected, the apparent average stiffness of the shorter specimens was lower than that observed for the longer specimens; in fact, compared to the $200-\mathrm{mm}$ specimen, the $150-\mathrm{mm}$ specimen resulted to be $5.95 \%$ less stiff, and the $100-\mathrm{mm}$ one $12.04 \%$ less stiff. This is in concert with the results reported for the GLARE, in that the flexibility of the specimen influences the results' quality. This can also be an explanation of why the values of bending stiffness found using GrindoSonic's data resulted in being higher than the one measured by 3-point bending test (refer to Table 2). Therefore, it is of interest to further develop the understanding of the vibrational behavior of such composites for obtaining more accurate results.

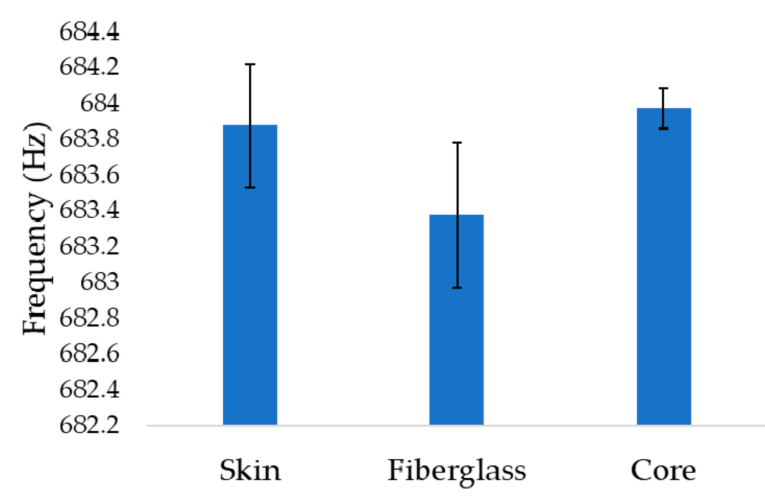

Figure 9. Frequency recorded by the GrindoSonic with probe placed in touch with different constituents of the 3D-FML specimens.

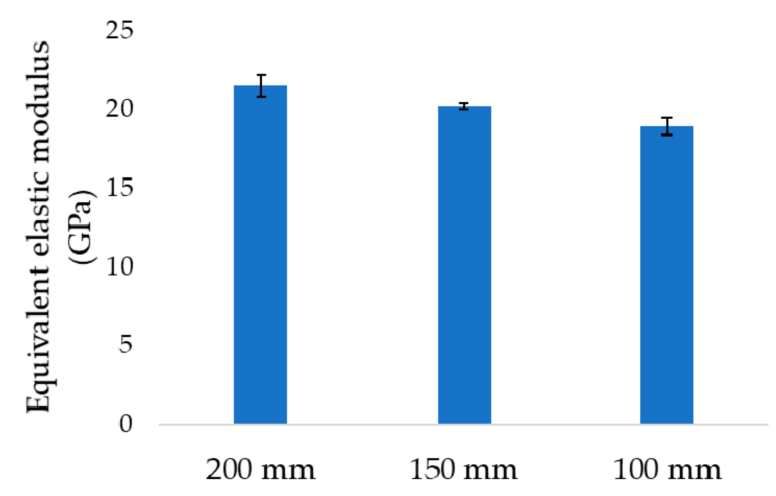

Figure 10. Estimated equivalent modulus of elasticity from GrindoSonic, for different 3D-FML specimen's lengths.

\section{Conclusions}

GrindoSonic (or sonic technique) was proved to be a reliable and accurate technique for establishing the vibration characteristics of various materials. The damping coefficients of aluminum, FRP, 3D-FML, and GLARE were measured and compared, revealing the superior damping capacity of the 3D-FML composite. Moreover, the results reported in this paper show that the equivalent material properties measured using this simple technique can be used to establish the flexural stiffness of a material system in an accurate and reproducible manner, regardless of the complex configuration of a given hybrid material. Finite element models were also developed to validate the accuracy of the findings and to show that the input of equivalent properties into FE models would lead to reliable results when simulating the dynamic response of materials. However, it was also shown that 
an excessively slender aspect ratio of a specimen causes an inaccurate frequency value to be reported by the GrindoSonic.

In addition, it is postulated that this technique could be used effectively and reliably to detect the presence of damage (e.g., fiber fracture, delamination, matrix cracking, longitudinal matrix splitting, debonding, and fiber pull-out) in a material system. In some of our other studies, we demonstrated how the change in vibration characteristics of a system can be used in conjunction with an effective signal processing technique (such as the Empirical Modal Decomposition), to reliably establish the presence and extent of damage, non-destructively [42-44]. Such a technique can also be used to identify difference in fiber properties, as each fiber used in composites are unique in their properties. Furthermore, the properties of the fibers vary based on the manufacturing methods and types, and these changes could be quantified using the impulse excitation technique. Our future work will focus on these important engineering challenges.

Acknowledgments: The authors acknowledge the financial support provided by the National Sciences and Engineering Research Council of Canada (NSERC) and the MITACS Globalink fellowship program. The authors are indebted to these agencies. The authors would also like to thank Laliberté for kindly providing the GLARE panel.

Author Contributions: Davide De Cicco performed the experiments, analyzed the data and wrote the draft paper. Farid Taheri provided the idea, directed the research and edited the paper.

Conflicts of Interest: The authors declare no conflict of interest.

\section{Appendix}

Table A1. Material properties used for the finite element simulations.

\begin{tabular}{|c|c|c|c|c|c|c|c|}
\hline \multirow{2}{*}{ Property } & \multirow{2}{*}{$\begin{array}{l}\text { FRP and GLARE } \\
\text { Fiberglass Plies }\end{array}$} & \multirow{2}{*}{$\begin{array}{l}\text { GLARE } \\
\text { Aluminum }\end{array}$} & \multicolumn{4}{|c|}{ 3D-FML } & \multirow{2}{*}{ Aluminum } \\
\hline & & & Glass-Epoxy Plies & Glass-Epoxy Pillars & Magnesium & Foam & \\
\hline$\rho\left(\mathrm{kg} / \mathrm{m}^{3}\right)$ & 1630 & 2700 & 1630 & 1630 & 1740 & 0.128 & 2676 \\
\hline$E_{11}$ or $E(G P a)$ & 25 & 72.4 & 9 & 3 & 36 & 0.05 & 68.9 \\
\hline $\mathrm{E}_{22}(\mathrm{GPa})$ & 6 & & 9 & 1 & & & \\
\hline $\mathrm{E}_{33}(\mathrm{GPa})$ & 6 & & 1 & 1 & & & \\
\hline $\mathrm{v}_{21}$ or $\mathrm{V}$ & 0.06 & 0.33 & 0.25 & 0.25 & 0.35 & 0 & 0.33 \\
\hline $\mathrm{v}_{31}$ & 0.06 & & 0.028 & 0.083 & & & \\
\hline $\mathrm{v}_{32}$ & 0.25 & & 0.028 & 0.083 & & & \\
\hline $\mathrm{G}_{12}=\mathrm{G}_{13}(\mathrm{GPa})$ & 1 & & 1 & 1 & & & \\
\hline $\mathrm{G}_{23}(\mathrm{GPa})$ & 2.4 & & 3.6 & 0.4 & & & \\
\hline
\end{tabular}

\section{References}

1. Lau, K.T.; Hung, P.Y.; Zhu, M.H.; Hui, D. Properties of natural fibre composites for structural engineering applications. Compos. Part B Eng. 2018, 136, 222-233. [CrossRef]

2. Soutis, C. Fibre reinforced composites in aircraft construction. Prog. Aerosp. Sci. 2005, 41, 143-151. [CrossRef]

3. Golzar, M.; Poorzeinolabedin, M. Prototype fabrication of a composite automobile body based on integrated structure. Int. J. Adv. Manuf. Technol. 2010, 49, 1037-1045. [CrossRef]

4. Prince, K. Composites win over sports market. Reinf. Plast. 2002, 46, 48-51. [CrossRef]

5. Sfondrini, M.F.; Cacciafesta, V.; Scribante, A. Shear bond strength of fibre-reinforced composite nets using two different adhesive systems. Eur. J. Orthod. 2011, 33, 66-70. [CrossRef] [PubMed]

6. Cacciafesta, V.; Sfondrini, M.F.; Lena, A.; Scribante, A.; Vallittu, P.K.; Lassila, L. V Flexural strengths of fiber-reinforced composites polymerized with conventional light-curing and additional postcuring. Am. J. Orthod. Dentofac. Orthop. 2007, 132, 524-527. [CrossRef] [PubMed]

7. Noor, A.K. Free-Vibrations-of-Multilayered-Composite-Plates.pdf. Am. Inst. Aeronaut. Astronaut. 1972, 11, 1038-1039.

8. Nayak, A.K.; Moy, S.S.J.; Shenoi, R.A. Free vibration analysis of composite sandwich plates based on Reddy's higher-order theory. Compos. Part B Eng. 2002, 33, 505-519. [CrossRef]

9. Nilsson, E.; Nillson, A.C. Prediction and Measurement of Some Dynamic Properties of Sandwich Structures with Honeycomb and Foam Cores. J. Sound Vib. 2002, 251, 409-430. [CrossRef] 
10. Neves, A.M.A.; Ferreira, A.J.M.; Carrera, E.; Cinefra, M.; Roque, C.M.C.; Jorge, R.M.N.; Soares, C.M.M. Static, free vibration and buckling analysis of isotropic and sandwich functionally graded plates using a quasi-3D higher-order shear deformation theory and a meshless technique. Compos. Part B Eng. 2013, 44, 657-674. [CrossRef]

11. Kant, T.; Swaminathan, K. Analytical solutions for free vibration of laminated composite and sandwich plates based on a higher-order refined theory. Compos. Struct. 2001, 53, 73-85. [CrossRef]

12. Tu, T.M.; Thach, L.N.; Quoc, T.H. Finite element modeling for bending and vibration analysis of laminated and sandwich composite plates based on higher-order theory. Comput. Mater. Sci. 2010, 49, S390-S394. [CrossRef]

13. Sayyad, A.S.; Ghugal, Y.M. On the free vibration analysis of laminated composite and sandwich plates: A review of recent literature with some numerical results. Compos. Struct. 2015, 129, 177-201. [CrossRef]

14. Liu, A.; Wang, K.W.; Bakis, C.E. Effect of functionalization of single-wall carbon nanotubes (SWNTs) on the damping characteristics of SWNT-based epoxy composites via multiscale analysis. Compos. Part A Appl. Sci. Manuf. 2011, 42, 1748-1755. [CrossRef]

15. DeValve, C.; Pitchumani, R. Experimental investigation of the damping enhancement in fiber-reinforced composites with carbon nanotubes. Carbon N. Y. 2013, 63, 71-83. [CrossRef]

16. Khan, S.U.; Li, C.Y.; Siddiqui, N.A.; Kim, J.-K. Vibration damping characteristics of carbon fiber-reinforced composites containing multi-walled carbon nanotubes. Compos. Sci. Technol. 2011, 71, 1486-1494. [CrossRef]

17. DeValve, C.; Pitchumani, R. Analysis of vibration damping in a rotating composite beam with embedded carbon nanotubes. Compos. Struct. 2014, 110, 289-296. [CrossRef]

18. Araújo, A.L.; Martins, P.; Mota Soares, C.M.; Mota Soares, C.A.; Herskovits, J. Damping optimisation of hybrid active-passive sandwich composite structures. Adv. Eng. Softw. 2012, 46, 69-74. [CrossRef]

19. Trindade, M.A.; Benjeddou, A.; Ohayon, R. Piezoelectric Active Vibration Control of Damped Sandwich Beams. J. Sound Vib. 2001, 246, 653-677. [CrossRef]

20. Sargianis, J.J.; Kim, H.I.; Andres, E.; Suhr, J. Sound and vibration damping characteristics in natural material based sandwich composites. Compos. Struct. 2013, 96, 538-544. [CrossRef]

21. Prabhakaran, S.; Krishnaraj, V.; Senthil Kumar, M.; Zitoune, R. Sound and vibration damping properties of flax fiber reinforced composites. Procedia Eng. 2014, 97, 573-581. [CrossRef]

22. Sargianis, J.; Suhr, J. Core material effect on wave number and vibrational damping characteristics in carbon fiber sandwich composites. Compos. Sci. Technol. 2012, 72, 1493-1499. [CrossRef]

23. Sargianis, J.; Suhr, J. Effect of core thickness on wave number and damping properties in sandwich composites. Compos. Sci. Technol. 2012, 72, 724-730. [CrossRef]

24. Asaee, Z.; Shadlou, S.; Taheri, F. Low-velocity impact response of fiberglass/magnesium FMLs with a new 3D fiberglass fabric. Compos. Struct. 2015, 122, 155-165. [CrossRef]

25. Asaee, Z.; Mohamed, M.; De Cicco, D.; Taheri, F. Low-Velocity Impact Response and Damage Mechanism of 3D Fiber-Metal Laminates Reinforced with Amino-Functionalized Graphene Nanoplatelets. Int. J. Compos. Mater. 2017, 7, 20-36. [CrossRef]

26. Cheraghi, N.; Riley, M.J.; Taheri, F. Application of hilbert-huang transform for evaluation of vibration characteristics of plastic pipes using piezoelectric sensors. Struct. Eng. Mech. 2007, 25, 1-22. [CrossRef]

27. Naghipour, M.; Mehrzadi, M.; Taheri, F.; Zou, G.P. Polynomial correction function for half-power bandwidth (HPB) method of damping of glulam beams reinforced with e-glass reinforced epoxy polymer (GRP). Can. J. Civ. Eng. 2009, 36, 241-252. [CrossRef]

28. Viens, M.J.; Johnson, J.J. Determination of Elastic Moduli of Fiber Resin Composites Using an Impulse Excitation Technique; NASA Technical Memorandum 104629; NASA Goddard Space Flight Center: Greenbelt, MD, USA, 1996.

29. GrindoSonic: Applications for Composite Materials. Available online: http://www.grindosonic.com/ applications / composites_plastics.html (accessed on 15 February 2018).

30. Zou, G.P.; Naghipour, M.; Taheri, F. A nondestructive method for evaluating natural frequency of glued-laminated beams reinforced with GRP. Nondestruct. Test. Eval. 2003, 19, 53-65. [CrossRef]

31. Droz, C.; Bareille, O.; Ichchou, M.N. A new procedure for the determination of structural characteristics of sandwich plates in medium frequencies. Compos. Part B Eng. 2017, 112, 103-111. [CrossRef]

32. Tiwari, K.A.; Raisutis, R.; Samaitis, V. Hybrid Signal Processing Technique to Improve the Defect Estimation in Ultrasonic Non-Destructive Testing of Composite Structures. Sensors 2017, 17. [CrossRef] 
33. Asaee, Z.; Taheri, F. Enhancement of Performance of 3D Fiber Metal Laminates under Low Velocity Impact-A coupled Numerical and Experimental Investigation. J. Sandw. Struct. Mater. 2017. [CrossRef]

34. Asaee, Z.; Taheri, F. Experimental and numerical investigation into the influence of stacking sequence on the low-velocity impact response of new 3D FMLs. Compos. Struct. 2016, 140, 136-146. [CrossRef]

35. Asundi, A.; Choi, A.Y.N. Fiber metal laminates: An advanced material for future aircraft. J. Mater. Process. Technol. 1997, 63, 384-394. [CrossRef]

36. De Cicco, D.; Taheri, F. Delamination Buckling Response of 3D Fiber-Metal Laminates Subjected to Different Loading Rates. In Proceedings of the American Society for Composites: Thirty-First Technical Conference; DEStech Publications, Inc.: Williamsburg, VA, USA, 2016; p. 12.

37. GrindoSonic. Operating Instructions for the GrindoSonic MK5i Instrument; JW Lemmens: Leuven, Belgium; pp. 1-23.

38. Kelly, S.G. Fundamentals of Mechanical Vibrations; McGraw-Hill Education: New York, NY, USA, 1992.

39. Yang, X.; Ji, S.; Song, L. Signal Analysis and Processing Platform Based on LabVIEW. Sens. Tansducers 2014, 172, 165-171.

40. Braem, M.; Lambrechts, P.; Van Doren, V.; Vanherle, G. The impact of composite structure on its elastic response. J. Dent. Res. 1986, 65, 648-653. [CrossRef] [PubMed]

41. Schultz, A.B.; Tsai, S.W. Dynamic Ratios in Damping Fiber-Reinforced Composites. J. Compos. Mater. 1968, 2, 368-379. [CrossRef]

42. Razi, P.; Esmaeel, R.A.; Taheri, F. Improvement of a vibration-based damage detection approach for health monitoring of bolted flange joints in pipelines. Struct. Heal. Monit. 2013, 12, 207-224. [CrossRef]

43. Esmaeel, R.A.; Taheri, F. Application of a simple and cost-effective method for detection of bolt self-loosening in single lap joints. Nondestruct. Test. Eval. 2013, 28, 208-225. [CrossRef]

44. Rezaei, D.; Taheri, F. Damage identification in beams using empirical mode decomposition. Struct. Heal. Monit. 2010, 10, 261-274. [CrossRef]

(C) 2018 by the authors. Licensee MDPI, Basel, Switzerland. This article is an open access article distributed under the terms and conditions of the Creative Commons Attribution (CC BY) license (http:/ / creativecommons.org/licenses/by/4.0/). 